\title{
Orbitofrontal activation restores insight lost after cocaine use
}

\author{
Federica Lucantonio ${ }^{1,2}$, Yuji Takahashi ${ }^{1}$, Alexander F Hoffman ${ }^{1}$, Chun Y Chang ${ }^{1}$, Sheena \\ Chaudhary ${ }^{1}$, Yavin Shaham ${ }^{1}$, Carl R Lupica ${ }^{1,{ }^{*}}$, and Geoffrey Schoenbaum ${ }^{1,2,3,{ }^{*}}$ \\ ${ }^{1}$ NIDA-IRP, 251 Bayview Boulevard, Baltimore, MD 21224 \\ ${ }^{2}$ Department of Anatomy and Neurobiology, University of Maryland School of Medicine, 20 Penn \\ St, HSF-2 S251, Baltimore, MD 21201
}

${ }^{3}$ Solomon H. Snyder Department of Neuroscience, The Johns Hopkins University, Baltimore, MD 21287

\section{Abstract}

\begin{abstract}
Addiction is characterized by a lack of insight into the likely outcomes of one's behavior. Insight or the ability to imagine outcomes is evident when outcomes have not been directly experienced. Using this concept, work in both rats and humans has recently identified neural correlates of insight in the medial and orbital prefrontal cortices. Here we show that these correlates are selectively abolished in rats by cocaine self-administration. Their abolition was associated with behavioral deficits and reduced synaptic efficacy in orbitofrontal cortex, reversal of which by optogenetic activation restored normal behavior. These results provide a link between cocaine use and problems with insight. Deficits in these functions are likely to be particularly important for problems such as drug relapse, in which behavior fails to account for likely adverse outcomes. As such, these data provide a neural target for therapeutic approaches to address these defining longterm effects of drug use.
\end{abstract}

Drug addiction is characterized by an inability to correctly adapt behavior in a changing environment ${ }^{1}$. Studies in humans have suggested that this reflects a lack of insight ${ }^{2-4}$. Operationalized, insight requires the ability to mentally simulate the causes and likely outcomes of one's behavior. The ability to mentally simulate or imagine likely outcomes can be revealed in situations where the outcomes have not been directly experienced previously. Using this concept, work in rats, monkeys, and humans has recently identified neural correlates of insight in the medial and orbital prefrontal cortices ${ }^{5-8}$.

\footnotetext{
Users may view, print, copy, and download text and data-mine the content in such documents, for the purposes of academic research, subject always to the full Conditions of use:http://www.nature.com/authors/editorial_policies/license.html\#terms

Correspondence and requests for materials should be addressed to F.L. (federica.lucantonio@ nih.gov) or G.S.

(geoffrey.schoenbaum@nih.gov).

These authors contibuted equally to this work as described in the author contributions.

Supplemental Information is available on the Nature Neuroscience web site.

Author Contributions: F.L. and G.S. conceived the behavioral and single-unit experiments with input from Y.S. and Y.T., F.L. and C.L. conceived the slice physiology work with input from A.H., and F.L. carried out the experiments, with assistance from Y.T., A.H., C.C., and S.C. F.L. analyzed the data, with assistance from Y.T., A.H., C.L., and G.S. The manuscript was prepared by F.L. and G.S with input from the other authors.

Reprints and permissions information is available at www.nature.com/reprints.

The authors declare no competing financial interests.
} 
Here we show that such neural correlates are selectively abolished in rats by prior use of cocaine. Their abolition was associated with specific deficits in insight-dependent behavior and also with a drug-induced reduction in the efficacy of glutamatergic neurotransmission at orbitofrontal pyramidal neurons, reversal of which by optogenetic activation restored normal behavior. These results provide a causal link between cocaine use and deficient insight. Deficits in such a fundamental building block underlying behavioral control across species 9,10 could be particularly important for problems such as drug relapse, in which behavior fails to account for likely outcomes, often despite apparent knowledge ${ }^{11-13}$. As such, these data provide a neural target for therapeutic approaches to address these vexing long-term effects of drug use.

\section{RESULTS}

\section{Prior cocaine self-administration disrupts insight, learning and associated neural correlates in the OFC}

Rats were trained $(\mathrm{n}=8)$ to self-administer cocaine for 3 -h/day for 14 days using a fixed ratio 1,40 -sec timeout schedule of reinforcement that has been associated with cardinal behaviors, such as relapse and incubation of cocaine craving, thought to be important models of addiction ${ }^{14}, 15$; control rats $(\mathrm{n}=12)$ were trained to respond for oral sucrose (a potent non-drug reward in rodents) using identical procedures (Fig. S1a-b). Three weeks after the end of self-administration, we recorded single-unit activity from the orbitofrontal cortex (OFC) in these rats as they were trained in a Pavlovian over-expectation task ${ }^{16}$, used previously to study orbitofrontal function ${ }^{6,17}$ (Fig. 1a and Table S1). This task consists of three phases: initial conditioning, compound training, and extinction testing. In initial conditioning, rats are trained that each of several different cues predicts the same amount of reward in the same location. In subsequent compound training, two of the cues are presented together, still followed by the same reward. Normal rats show increased responding to this compound cue - termed summation - that is thought to reflect a novel and immediate expectation for increased reward. As operationalized above, this increased expectation requires insight, because past experience does not directly dictate that greater reward should result when both cues occur simultaneously. Furthermore, because the compound cue yields the same reward as each individual cue, the novel expectation results in a prediction error and hence new learning, as evidenced by an immediate decline in responding to the one of the compounded cues when it is presented later, by itself, in the extinction test. Both summation and the resultant learning are dependent on the $\mathrm{OFC}{ }^{17}$, secondary to a sudden increase in activity in pyramidal neurons in this area during presentation of the compound cues ${ }^{6}$. These data suggest that the OFC generates the novel estimates and insight into likely outcomes upon which both summation and learning depend.

Conditioning (Fig. 1a) consisted of presentations of three auditory cues (A1, A2 and A3, counterbalanced) and a visual cue (V), each of which was paired with three sucrose pellets, except A3, which served as a CS-. Sucrose and cocaine-trained rats showed similar increases in conditioned responding to A1, A2 and V across sessions (Fig. S1c-d), and a similar increase in the proportion of single units responding to the cues, with the proportion of neurons showing a phasic increase in firing to at least one of the cues increasing to about 
$50 \%$ in both groups by the end of training (Fig. S1e-f). Moreover, there were no differences in baseline firing rates between groups in any session or neuron grouping (Table S2 and Fig. S2).

At the end of conditioning, rats were trained in a compound probe session (CP in Fig. 1a). This session consisted of additional conditioning (CP 1/2) followed by compound training (CP 2/2), in which A1 and V were presented concurrently (A1V) followed by the same reward as in initial conditioning. A2, A3 and $\mathrm{V}$ were presented individually throughout compound training, followed by the same reward, as in initial conditioning. The sucrosetrained rats showed a significant increase in responding to $\mathrm{A} 1$ when it was presented in compound with V (Fig. 2a). The increase was evident over the entire session, and also when only the first trial of compound training was considered (Fig. S3a). This effect was not evident in cocaine-trained rats, which responded the same amount to A1 alone and in compound with V (Figs. 2b and S3b). Notably, the increased responding to the A1V compound cue in the sucrose-trained group was specific to the compound cue; neither group showed any change in responding to the $\mathrm{A} 2$ control cue between the two phases.

We recorded 58 neurons from OFC in sucrose-trained rats and 131 neurons from OFC in cocaine-trained rats during the compound probe session. These populations included 33 in the sucrose group and 71 in the cocaine-trained group that exhibited an excitatory phasic response to at least one of the cues during the conditioning phase. Consistent with the idea that increased activity in OFC underlies increased responding to the compound cue, summation at the start of compound training in the sucrose-trained group was accompanied by a sudden increase in the phasic neural response to the compound cue in these neurons. This was evident in the population response, which was similar for A1, A2 and V during the conditioning phase, but increased selectively to A1V at the start of compound training (Figs. $2 \mathrm{c}$ and $2 \mathrm{e}$ ). The increase was evident over the entire session, and also when only the first trial of compound training was considered (Fig. S3c-e). This normal effect of compounding on neural firing in OFC was completely absent in the cocaine-trained rats (Figs. 2d,2f, and S3d-f). Moreover, no significant differences were found in neurons that exhibited an inhibitory response to at least one of the cues during the conditioning phase in both groups (Fig. S7).

The contrast between sucrose and cocaine-trained rats was also evident in index scores, capturing the change in neural activity in each cue-responsive neuron to A1 and A2 between conditioning and compound training. In the sucrose group, the distribution of these index scores shifted significantly above zero for A1 but not for A2 (Fig. 2g-h), whereas in the cocaine group, the distribution of the index scores did not shift for either cue (Fig. 2i-j). This pattern was also observed when only the first trial of compound phase was considered (Fig. S3 g-h for sucrose-trained rats; Fig. S3 i-j for cocaine-trained rats) and it was evident in both $\mathrm{A} 1$ and $\mathrm{V}$ preferring neurons (Fig. $\mathrm{S} 3 \mathrm{k}-\mathrm{l}$ ). The shift in firing to the A1 cue in sucrose group was directly correlated with increased conditioned responding to the compound cue (Fig. 2k), confirming that neural summation in OFC predicted behavioral summation in the sucrose-trained rats. No correlation was found in cocaine-trained rats (Fig. 21). 
Importantly the spontaneous increase in firing to the A1V compound observed in the sucrose group was not simply a reflection of the increased sensory input associated with the sudden combination of the two cues, but rather tracked the elevated expectations of reward. This was evident in a trial-by-trial analysis of activity in response to A1 and A2 within the first compound session; while activity to A2 was stable across trials, activity to A1 was highest on the first trial and then declined (Fig. 2m, inset). A similar pattern was evident in a comparison of the activity to $\mathrm{A} 1$ and $\mathrm{A} 2$ in OFC neurons recorded in the same locations in later compound sessions (Fig. 2m). By contrast, in the cocaine group, activity to A1 and A2 remained similar and was stable from the end of conditioning to the last compound session (Fig. 2n).

At the end of compound training, the rats were tested in an extinction probe session (PB in Fig. 1a). This session consisted of additional compound training (PB 1/2) followed by extinction training (PB 2/2), in which $\mathrm{A} 1$ and the other auditory cues were presented alone without the food reward. As expected, in the sucrose group, when A1 was separated from V at the start of extinction, the rats showed a sudden and selective decline in responding, which persisted throughout the extinction phase (Fig. 3a). Importantly, the reduction in responding to $\mathrm{A} 1$ was evident on the first trial of extinction. By contrast, in the cocainetrained group, the conditioned responding to A1 did not change when A1 was separated from $\mathrm{V}$ at the start of extinction. Instead these rats continued to respond at high levels to both $\mathrm{A} 1$ and $\mathrm{A} 2$ at the start of extinction (Fig. 3b). Interestingly, prior cocaine exposure did not impair extinction learning driven by omission of reward within the probe session itself; indeed, the cocaine-trained rats showed somewhat stronger extinction learning as evidenced by the more rapid decline in responding to $\mathrm{A} 1$ and $\mathrm{A} 2$ in the first few non-rewarded trials.

We recorded 96 neurons from OFC in sucrose-trained rats and 128 neurons from OFC in cocaine-trained rats during the extinction probe session, including 50 in the sucrose group and 69 in the cocaine group exhibiting an excitatory phasic response to at least one of the cues. In both groups, firing in response to A1V and A2 was statistically similar at the end of compound training, (PB 1/2, Fig. 3c-f). However, in the sucrose group, the firing spontaneously declined at the start of extinction training to A1. A direct comparison revealed a significant reduction of firing on the $1^{\text {st }}$ trial of the probe phase compared to firing in the compound phase for A1 (Fig. 3c) but not for A2 (Fig. 3e). Neurons recorded from cocaine-trained rats showed no significant change in firing (Fig. 3d-f). Moreover, no significant differences were found in neurons that exhibited an inhibitory response to at least one of the cues during the conditioning phase in both groups (Fig. S8).

A similar contrast was evident in the distribution of index scores comparing firing of each neuron to $\mathrm{A} 1$ and $\mathrm{A} 2$ at the end of compound training versus the $1^{\text {st }}$ trial in extinction. In the sucrose group, the distribution of these scores was shifted significantly below zero for A1 but not for A2 (Fig. 3g-h), and the shift in firing to the A1 cue on the $1^{\text {st }}$ trial of extinction was directly correlated with reduced responding shown by the rat in that session (Fig. 3k). Notably, reduced behavioral responding to A1 was inversely correlated with neural summation measured earlier in the first compound training session (Fig. $3 \mathrm{~m}$ ). Thus, the stronger the neural response to the compound cue was at the start of compound training, the weaker conditioned responding to the A1 cue was at the start of extinction. In the cocaine 
group, the distribution of the index scores did not shift for either cue (Fig. 3i-j), and there were no correlations between conditioned responding and neural activity (Fig. 31-3m). Thus, neural estimates of outcomes in OFC were predictive of both behavior and learning in rats that had self-administered sucrose (and also in naïve controls in our prior study ${ }^{6}$ ) but not in rats that had self-administered cocaine.

\section{Changes in insight and learning after cocaine self-administration are associated with reduced synaptic efficacy in OFC}

The neural data described above suggest that elevated activity in OFC during the compound cue is critical for learning and that the lack of this neural summation in OFC may explain the behavioral deficit seen after cocaine self-administration. Neural summation did not reflect ongoing learning. It did not have to be acquired. It manifested and in fact was greatest on the very first exposure to the compounded cues. Thus it reflected processing of existing information, presumably due to the reactivity of OFC networks to afferent input. One potential explanation for the loss of this spontaneous reactivity after cocaine selfadministration would be reduced synaptic efficacy within the OFC, particularly in the pyramidal cell population likely sampled by our recording electrodes. Such changes have been reported previously after drug use in corticolimbic circuits, including prefrontal regions ${ }^{12,18-23}$. To test this hypothesis, we conducted whole cell recordings from pyramidal neurons in brain slices containing the OFC, obtained from rats that had been behaviorally characterized after self-administration of either sucrose or cocaine (Fig. 4a). Rats in the two groups (N's = 9) exhibited behavioral effects that were similar to those described above, with sucrose-trained rats showing robust learning as result of compound training and cocaine-trained rats showing no effect (Fig. 4b-c; see Fig. S4 for full details on behavior). We measured glutamate-mediated miniature excitatory post-synaptic currents (mEPSCs) in voltage clamped $\left(\mathrm{V}_{\mathrm{m}}=-70 \mathrm{mV}\right)$ OFC pyramidal neurons in which inhibitory post-synaptic currents were blocked with the $\mathrm{GABA}_{\mathrm{A}}$ receptor antagonist picrotoxin (100 $\mu \mathrm{M})$ and sodium channels were blocked by tetrodotoxin (TTX, $0.5 \mu \mathrm{M}$ ). Consistent with a decline in synaptic efficacy, OFC pyramidal neurons from cocaine-trained rats showed a specific reduction in mEPSC frequency relative to sucrose-trained rats; mEPSC amplitudes and rise and decay times did not differ between groups (Fig. 4d-f). The cocaine-sensitive measure of excitability -mEPSC frequency - was also related to learning (Fig. 4g).

\section{Transient optogenetic activation of OFC neurons restores insight and learning lost after cocaine self-administration}

Previously we have shown that optogenetically inhibiting OFC activity during the compound cue prevents learning ${ }^{6}$. Given the reduction in the synaptic efficacy at pyramidal neurons in OFC, observed both in vivo and ex vivo in cocaine-trained rats, we conducted a final experiment to test whether artificially activating OFC pyramidal neurons would restore normal learning in cocaine-trained rats (Fig. 5a). Rats received bilateral infusions of either AAV-CaMKIIa-ChR2-eYPF ( $\mathrm{N}=9$ ) or AAV-CaMKII-eYFP (control, $\mathrm{N}=8$ ) into the OFC and had fiber optic assemblies implanted immediately above the injection sites. The CaMKII promoter was used to target cortical neurons within the $\mathrm{OFC}^{24,25}$; expression and lightdependent activation of OFC neurons was later confirmed using ex vivo recording (Fig. 5cd). 
These rats were trained to self-administer cocaine and then tested in the Pavlovian overexpectation task. Procedures were identical to those used earlier, except that $473 \mathrm{~nm}$ light was delivered into OFC bilaterally, time-locked to presentation of the compound cue. While there were no significant differences in the behavior of the eYFP and ChR2 rats during cocaine self-administration, conditioning or compound training (Fig. S5), ChR2 rats responded much less to A1 than to A2 in the probe test (Fig. 5e), whereas eYFP rats, lacking the critical opsin, failed to show any difference in conditioned responding to A1 versus A2 (Fig. 5f). The difference in the $\mathrm{ChR} 2$ rats reflected a significant decline in responding to $\mathrm{A} 1$ between compound training and the first trial of the extinction probe test. By contrast, in the eYFP group, conditioned responding to A1 did not change when it was separated from $\mathrm{V}$ at the start of extinction. Thus, cocaine-trained rats were again unable to integrate reward expectations to drive learning, and this deficit was reversed by optogenetic activation of pyramidal neurons in OFC during the compound cue.

Optogenetic activation of OFC might cause lasting changes in the response properties of stimulated neurons ${ }^{26-29}$. Indeed, long-lasting changes might even be desirable if one hopes to restore normal behavior after cocaine use. To test this possibility, we retrained the rats in the eYFP and ChR2 groups used in the prior experiment, and repeated the compound conditioning, this time delivering light into OFC during the inter-trial interval after each compound trial. Although activity in OFC during this period is not normally necessary for learning ${ }^{6}$, this pattern of activation was sufficient to restore normal learning in ChR2 (Fig. $5 \mathrm{~g}$ ) but not eYFP rats (Fig. 5h). These data suggest that brief, coordinated, and strong activation of OFC pyramidal neurons may have a prolonged effect on cocaine-induced changes in synaptic efficacy and information processing in OFC.

Of course, one interpretation of these results is that optogenetic activation of OFC simply masks the underlying deficit caused by cocaine by serving as a novel reward or a conditioned reinforcer or by altering the motivation or salience or "value" of the cues when it is delivered. While it is difficult to completely rule out these possibilities, the efficacy of stimulation during the inter-trial interval period, several minutes removed from the compound cues, would seem to make a direct rewarding effect unlikely. In addition, these mechanisms cannot easily account for the actual pattern of results. This is because any mechanism that does not augment predictions about the location and amount of food to be delivered would both compete with the conditioned food cup response during the compound cue, thus lowering it, and likely retard rather than promote extinction. It is only if the activation of the OFC in the context of the cues augments the prediction of the food pellets that one would expect extinction learning. Nevertheless, in order to directly test this hypothesis, we conducted an additional experiment in which ChR2 and eYFP rats were given the opportunity to press a lever to activate OFC using the same stimulation parameters employed above. We found that even after several days of training, neither group distinguished between this lever and a control (Fig. S6). These data show that the optogenetic activation of OFC using parameters sufficient to correct the learning deficit caused by cocaine is not sufficiently rewarding to support self-administration. 


\section{DISCUSSION}

Here we have shown that cocaine self-administration causes a long lasting deficit in the ability of rats to use insight into likely outcomes to guide behavior and engage learning mechanisms. This deficit reflected the loss of neural integration of outcome expectancies in the $\mathrm{OFC}$, an area generally thought to be important for signaling information about outcomes ${ }^{7,30-35}$. Although these changes in single unit correlates could reflect effects of cocaine on afferent structures, the brain slice data and the effects of optical stimulation in the ITI period argue against this interpretation. Specifically while the efficacy of stimulation might reflect a compensatory change or effects of local stimulation of OFC back propagating to impact the afferent region, this is much more difficult to envision for ITI stimulation than for stimulation during the actual cue. A more parsimonious explanation is that the local stimulation during the ITI corrects or at least overcomes or compensates for a local change. The brain slice data corroborate this account, since there is in fact a local effect to be corrected, in the form of the reduced frequency of neurotransmitter release from glutamatergic terminals within OFC. This change was related to earlier behavioral changes and was observed under conditions in which OFC is clearly isolated from other brain structures that might contribute to the behavior. While these terminals could arise from afferent, inputs or they may reflect localized OFC circuitry. Lastly it is also worth noting that cocaine and other psychostimulants disrupt performance (and neural correlates) in a variety of tasks that depend on the $\mathrm{OFC}^{36}$. While any one deficit might result from changes in an afferent brain area, the overall pattern is difficult to reconcile with a single effect elsewhere. Thus, overall, these findings fit best with a model of addiction in which exposure to some addictive drugs causes a critical loss of this function in the OFC $13,36,37$. This model is consistent with data from human addicts and animal models of addiction showing alterations in OFC and in OFC-dependent tasks $21,38-46$.

Importantly, the effect of cocaine on over-expectation did not reflect a general learning impairment; sucrose- and cocaine-trained rats acquired a conditioned response to the food cup at the same rate and of the same intensity, and neurons in OFC responded similarly to the directly experienced Pavlovian cues in both groups. Moreover, the same rats that were unable to extinguish responding during over-expectation were able to extinguish responding when the actual reward was omitted. Thus cocaine-trained rats did not suffer from a general deficit in response inhibition or extinction learning. In this regard, it is important to point out that extinction in response to over-expectation shows spontaneous recovery and other phenomena that are the hallmarks of extinction by omission ${ }^{47-49}$. It differs only in that it requires the subject to mentally simulate or imagine a novel reward for generation of the negative prediction error that drives learning, whereas extinction by reward omission does not. This subtle distinction provides potential insight into the puzzling inability of human addicts to effectively extinguish drug-seeking behavior in complex, real-world environments, where accurate predictions are very likely to involve mental simulation and the integration of predictions from multiple sources. Hence, these findings may have important implications for understanding the neural circuitry critical to the loss of behavioral control that characterizes addiction. 
Our results also have potential therapeutic relevance. The behavioral deficit was associated with reduced pyramidal neuron excitability in OFC in vivo and in vitro, the reversal of which by activation of pyramidal neurons at the time of the compound cue reversed the behavioral deficit. Further, artificial activation of the pyramidal neurons during the intertrial interval - a period during which inhibition of OFC has no effect on behavior in this task ${ }^{6}$ also rescued the cocaine-induced learning deficit. This finding implies that the required information is latent in the network or is afferent at the time of learning, and that OFC must only be put back "online" to restore normal function. In favor of this, activation of OFC did not impact other behavioral measures, such as the responding to the control cues or even the later extinction by reward omission.

This selective improvement holds the promise that it may be possible to selectively mitigate the effects of drug use on OFC function (and perhaps the function of other prefrontal regions ${ }^{20}$ ) by transiently activating the network in a coordinated but temporally nonspecific fashion. The brief but coordinated period of strong activity may at least temporarily increase the excitability of the network ${ }^{26}$, thereby restoring normal function. This raises the possibility that brief, coordinated activation of this brain area -either once or on an ongoing basis - might represent a viable therapeutic approach that would improve OFC-dependent insight in addicts.

\section{ONLINE METHODS}

\section{Subjects}

Male Long-Evans rats (Charles River Labs) weighing 250-275 g upon arrival were housed individually on a 12 hour light/dark schedule (lights on at 7:00 A.M.). All rats were given free access to food except during testing periods. During behavioral testing (selfadministration and over-expectation training), rats were food deprived to $85 \%$ of their baseline weight, by giving $10 \mathrm{~g}$ of pellets each day until the desired weight was reached and then maintained at $85 \%$ with $15 \mathrm{~g}$ of pellets per day. Water was freely available throughout the experiments. No statistical test was run to determine sample size a priori. The sample sizes we chose are similar to those used in previous publications. All testing was conducted at the National Institute on Drug Abuse Intramural Research Program in accordance with NIH guidelines.

\section{Catheter Surgery}

All rats were implanted with a chronically indwelling intravenous catheter. Rats were anaesthetized with ketamine (100 mg/ kg, i.p., Sigma) and xylazine (10 mg/kg, i.p., Sigma). A silastic catheter was inserted into the right jugular vein and passed subcutaneously to the back, where it was attached to a modified 22-gauge cannula (Plastics One) and fixed to the rat's back with sutures. Carprofen $(0.1 \mathrm{mg} / \mathrm{kg}$, s.c., Pfizer) was given after surgery as an analgesic. Rats recovered for 7-10 days before starting behavioral testing. During recovery and self-administration training, catheters were flushed every day with sterile $0.9 \%$ saline + the antibiotic Gentamicin $(0.08 \mathrm{mg} / \mathrm{mL}$, BioSource International). 


\section{Stereotaxic Surgeries}

For the single unit recording experiment, drivable bundles of 10-25-um diameter $\mathrm{FeNiCr}$ recording electrodes (Stablohm 675, California Fine Wire) were implanted unilaterally in OFC under stereotaxic guidance at $3.0 \mathrm{~mm}$ anterior and $3.2 \mathrm{~mm}$ lateral to bregma and 4.2 $\mathrm{mm}$ ventral to the brain surface. Electrodes were advanced subsequently into final positions within OFC during recording.

For the optogenetic experiment, AAV-CaMKIIa-eChR2-eYFP or AAV-CaMKIIa-eYFP (from Gene Therapy Center at University of North Carolina at Chapel Hill, courtesy of Dr. Karl Deisseroth) was injected bilaterally in OFC under stereotaxic guidance at $3.0 \mathrm{~mm}$ anterior and $3.2 \mathrm{~mm}$ lateral to bregma and 4.4 and $4.5 \mathrm{~mm}$ from the brain surface. A total 1$1.2 \mu \mathrm{l}$ of virus (titer $\sim 10^{12}$ ) per hemisphere was delivered at the rate of $\sim 0.1 \mu \mathrm{l} / \mathrm{min}$ by Picosptrizer microinjection system (Parker, Hollins, NH). Optic fibers $(200 \mu \mathrm{m}$ in core diameter; Thorlab, Newton, NJ) were implanted bilaterally at $3.0 \mathrm{~mm}$ anterior and $3.2 \mathrm{~mm}$ lateral to bregma and $4.2 \mathrm{~mm}$ ventral to the brain surface.

\section{Self-Administration (SA)}

Following recovery from surgery, the rats were allowed to self-administer cocaine- $\mathrm{HCl}$ $(0.75 \mathrm{mg} / \mathrm{kg} / \mathrm{infusion} ; \mathrm{n}=34)$, or sucrose $(10 \% \mathrm{w} / \mathrm{v} ; \mathrm{n}=21)$ for 14 consecutive days. Study groups were blinded. Cocaine hydrochloride (NIDA, Bethesda, MD) was dissolved in sterile $0.9 \%$ saline.

Rats were trained in standard behavioral chambers purchased from Coulbourn Instruments, each enclosed in a sound-resistant shell. Each chamber was equipped with two levers. The two levers were on opposite walls and $8 \mathrm{~cm}$ from the grid floor. For drug selfadministration, silastic tubing shielded with a metal spring extended from each animal's intravenous catheter to a liquid swivel (Instech Laboratories, Plymouth Meeting) mounted on an arm fixed outside of the operant chamber. Tygon tubing extended from the swivel to an infusion pump (Med Associates Inc) located adjacent to the external chamber. For sucrose training, a dipper was recessed in the center of one end wall. Entries were monitored by photobeam.

Rats were trained to self-administer cocaine- $\mathrm{HCl}$ under a fixed ratio 1 (FR1) schedule of reinforcement, such that every press on the active lever delivered a $4 \mathrm{sec}$ infusion of cocaine. "Priming" injections were never given. Daily SA sessions lasted 3 h, with 15-min timeout periods after each hour. Each session began with the insertion of the active lever. Each infusion was accompanied by the retraction of the active lever and followed by a 40 -sec timeout period in which the lever was inserted again. Pressing on the inactive lever had no programmed consequences. At the end of each session the active lever was retracted. The number of cocaine infusions was limited to $20 / \mathrm{h}$ to prevent overdose. After 20 infusions, the active lever was retracted for the remainder of the hour. For sucrose SA, procedures were the same as those for cocaine SA, except that rats were shaped to retrieve sucrose, and active lever presses resulted in the insertion into the chamber recess of a dipper containing a 0.05 $\mathrm{ml}$ of a $10 \%$ sucrose solution for $4 \mathrm{sec}$. 


\section{Pavlovian Over-expectation training}

Three weeks after the end of self-administration training, rats began Pavlovian overexpectation training. Rats designated for single unit recording experiments received the training in aluminum chambers approximately 18 inches on each side with sloping walls narrowing to an area of $12 \times 12$ inches at the bottom. Rats designated for optogenetic and ex vivo electrophysiology experiments received the training in behavioral chambers. In both cases, a food cup was recessed in the center of one end wall. Entries were monitored by photobeam. Two food dispensers containing $45 \mathrm{mg}$ sucrose pellets (banana- or grapeflavored; Bio-serv) delivered pellets to the food cup. White noise or a tone, each measuring approximately $76 \mathrm{~dB}$, was delivered via a wall speaker. A clicker $(2 \mathrm{~Hz})$ and a $6 \mathrm{~W}$ bulb were also mounted on that wall.

Rats were shaped to retrieve food pellets, and then underwent 10-12 conditioning sessions. In each session, the rats received eight $30 \mathrm{~s}$ presentations of three different auditory stimuli (A1, A2 and A3) and one visual stimulus (V). Each session consisted of 8 blocks, and each block consisted of 4 presentation of a cue. The order of cue-blocks was counterbalanced and randomized. For all conditioning, V consisted of a cue light, and A1, A2 and A3 consisted of a tone, clicker or white noise, respectively (counterbalanced). Two differently flavored sucrose pellets (banana and grape, designated as $\mathrm{O} 1$ and $\mathrm{O} 2$, counterbalanced) were used as rewards. $\mathrm{A} 1$ and $\mathrm{V}$ terminated with delivery of three pellets of $\mathrm{O} 1$, and $\mathrm{A} 2$ terminated with delivery of three pellets of O2. A3 was paired with no food. After completion of conditioning training, rats received a single session of compound probe $(\mathrm{CP})$. During the $1^{\text {st }}$ half of the session, the initial conditioning continued, with 6 trials each of 4 cues, in a blocked design, with order counterbalanced. During the $2^{\text {nd }}$ half of the session, compound training began with 6 trials of concurrent $\mathrm{A} 1$ and $\mathrm{V}$ presentation, followed by delivery of the same reward as during initial conditioning. A2, A3 and V continued to be presented as in initial conditioning, with 6 trials each stimulus. These cues were also presented in a blocked design with order counterbalanced. After the compound probe, rats received 3 days of compound training sessions $(\mathrm{CP} 2-\mathrm{CP} 4)$ with 12 presentations of $\mathrm{A} 1 \mathrm{~V}, \mathrm{~A} 2, \mathrm{~A} 3$ and $\mathrm{V}$. One day after the last compound training, rats received a single extinction probe session (PB). During the $1^{\text {st }}$ half of the session, the compound training continued with 6 presentations of A1V, A2, A3 and V. During $2^{\text {nd }}$ half of the session, rats received eight non-reinforced presentations of $\mathrm{A} 1, \mathrm{~A} 2$ and $\mathrm{A} 3$, with the order mixed and counterbalanced.

In rats designated for single-unit recording experiments, following the probe test, the electrode was typically advanced to a new location, and the rats repeated days 11 and 12 of conditioning and then underwent additional rounds of over-expectation training in order to acquire additional data. This was done up to two times for a given rat, resulting in 32 rounds of training for the sucrose group and 24 rounds of training for the cocaine group. Neural data from the initial compound and extinction days were not statistically different from data gathered in later rounds of training and thus these neurons were analyzed together in the text.

The primary measure of conditioning to cues was the percentage of time that each rat spent with its head in the food cue during the last 20-sec conditioned stimulus (CS) presentation, as indicated by disruption of the photobeam. We also measured the percentage of time that 
each rat showed rearing behavior during the last 20 -sec CS period. To correct for time spent rearing, the percentage of responding during the last 20-sec CS was calculate as follows: $\%$ of responding $=100 *([\%$ of time in food cup $]) /[100-(\%$ of time of rearing $)])$.

\section{Single-Unit recording}

Throughout the Pavlovian over-expectation training, rats were attached to the recording cable and prior to each session, wires were screened for activity. Active wires were selected for recording, and the session was begun. On the rare occasion that less than 4/8 wires were active, then the electrode assembly was advanced 40 or $80 \mathrm{um}$ at the end of the session. Otherwise the electrode was kept in the same position between sessions within a single round of over-expectation training. After the extinction probe test, ending a round of training, the electrode assembly was advanced 80 um regardless of the number of active wires in order to acquire activity from a new group of neurons in any subsequent training.

Neural activity was recorded using two identical Plexon Multichannel Acquisition Processor Systems (Dallas, TX), interfaced with training chambers described above. After amplification and filtering, waveforms ( $>$ 2.5:1 signal-to-noise) were extracted from active channels and recorded to disk by an associated workstation with event timestamps. Units were stored using Offline Sorter software from Plexon Inc (Dallas, TX), using a template matching algorithm. Sorted files were processed in Neuroexplorer to extract unit timestamps and relevant event markers and analyzed in Matlab (Natick, MA).

Firing activity in the last $20 \mathrm{~s}$ of each CS was compared to activity in the last $20 \mathrm{~s}$ of the preCS period by t-test $(\mathrm{p}<0.05)$. Neurons with significantly higher activity during at least one of the 4 cues were defined as "cue-responsive" as described in the main text. Normalized firing rate was calculated by dividing the mean firing rate during the last $20 \mathrm{sec}$ of CS by the mean firing rate in the last $20 \mathrm{sec}$ of pre-CS period.

\section{In vivo optical excitation sessions with $\mathrm{ChR2}$ during over-expectation}

Throughout the Pavlovian over-expectation training, rats were attached to fiber optic patch cables coupled to a solid state laser (473 nm; Laser Century, Shanghai, China) via an optic commutator (Doric Lenses, Quebec, Canada) and light (15 Hz, $473 \mathrm{~nm}, 10-15 \mathrm{~mW}$ ) was delivered into the OFC bilaterally during each compound session during the compound cue 50

\section{Freely moving optical self-stimulation}

A separate group of rats were injected with AAV-CaMKIIa-eChR2-eYFP $(\mathrm{N}=10)$ or AAVCaMKIIa-eYFP $(\mathrm{N}=7)$ into the OFC and implanted with chronic optic fibers above the OFC (see above for specific coordinates). At $21-28 \mathrm{~d}$ after virus injections, rats were mildly foodrestricted to facilitate behavioral responding. Immediately before placing rats in the operant chambers, rats were attached to fiber optic patch cables, coupled to a solid-state 473-nm laser outside the operant chamber. Operant chambers were equipped with one active and one inactive lever. A 1-h optical self-stimulation session began with the insertion of the two levers. Each active lever press performed by the animal resulted in an optical stimulation of 
OFC (15 Hz, $473 \mathrm{~nm}, 10-15 \mathrm{~mW})$ and lever retraction for 30 seconds. Both active and inactive lever press data were recorded.

\section{Slice preparation and ex vivo electrophysiology}

At the end of over-expectation training, rats were anesthetized with isoflurane and decapitated using a guillotine. Brains were removed and transferred to a beaker containing oxygenated (95\% O2/5\% CO2), ice-cold cutting solution (in mM: sucrose, 194; $\mathrm{NaCl}, 30$; $\mathrm{KCl}, 4.5 ; \mathrm{MgCl}_{2}, 1 ; \mathrm{NaH}_{2} \mathrm{PO}_{4}, 1.2$; glucose, $\left.10 ; \mathrm{NaHCO}_{3}, 26\right)$. Coronal sections ( $\left.250 \mu \mathrm{m}\right)$ were cut using a vibrating blade microtome (Leica VT1000). The slices were transferred to an oxygenated holding chamber filled with normal artificial cerebral spinal fluid (aCSF, in $\mathrm{mM}: \mathrm{NaCl}, 126 ; \mathrm{KCl}, 3 ; \mathrm{MgCl}_{2}, 1.5 ; \mathrm{CaCl}_{2}, 2.4 ; \mathrm{NaH}_{2} \mathrm{PO}_{4}, 1.2 ;$ glucose, $11 ; \mathrm{NaHCO}_{3}, 26$ ) at $34^{\circ} \mathrm{C}$ for 20 to 30 minutes, then the holding chamber was permitted to reach room temperature where it was maintained for the for the rest of the incubation period.

For recording, a single slice was transferred to a heated chamber $\left(31-33^{\circ} \mathrm{C}\right)$ and perfused with normal aCSF $(2 \mathrm{ml} / \mathrm{min})$ containing picrotoxin $(100 \mu \mathrm{M})$ and tetrodotoxin (TTX, $200 \mathrm{nM}$ ) using a peristaltic pump (Cole-Parmer). Visualization of OFC neurons was performed with an upright microscope equipped for epifluorescence and differential interference contrast (DIC) microscopy (BX51WI, Olympus, Japan). Recording electrodes $(\sim 5 \mathrm{M} \Omega$ ) were filled with (in $\mathrm{mM}$ ): K-gluconate, 140; KCl, 5; HEPES, 10; EGTA, 0.2; $\mathrm{MgCl} 2$, 2; Mg-ATP, 4; Na2-GTP, 0.3; Na2-phosphocreatine, 10; pH 7.2 with KOH. Wholecell clamp recordings were performed using an Axopatch 200B amplifier (Molecular Devices, Sunnyvale, CA). Cells were clamped at $-70 \mathrm{mV}$, and mEPSCs were sampled at 4$10 \mathrm{kHz}$ using WinLTP software (WinLTP Ltd, Bristol, UK) and an A/D board (National Instruments PCI-6251) residing in a personal computer. Optically elicited responses were elicited using a $473 \mathrm{~nm}$ laser (DPSSL, OEM Laser) delivered via a 200 um core fiber positioned near the surface of the slice. Hyperpolarizing $(-10 \mathrm{mV})$ voltage steps were delivered via the recording electrode every 30 s in order to monitor whole-cell access. Only cells with stable access ( $<10 \%$ change in resistance) were included in analyses. Analysis of mEPSCs was performed off-line using the MiniAnalysis program (v 6.0, Synaptosoft Inc., Fort Lee, NJ).

\section{Histology}

At the end of the single unit recording experiment, rats were deeply anesthetized and then the final electrode position was marked by the passage of a current though each microwire to create a small iron deposit. The rats were then perfused with 4\% PFA and potassium ferrocyanide solution to visualize the iron deposit. The brains were removed from the skulls and processed for histology using standard techniques.

At the end of the optogenetic experiment, rats with optic fibers were anesthetized with isofluorane and brains were processed as previously described in the slice preparation section. Expression of ChR2-eYFP was examined using a confocal microscope.

A supplementary methods checklist is available. 


\section{Supplementary Material}

Refer to Web version on PubMed Central for supplementary material.

\section{Acknowledgments}

This work was supported by funding from NIDA. The authors would like to thank Dr Karl Deisseroth and the Gene Therapy Center at the University of North Carolina at Chapel Hill core for providing viral reagents, and $\mathrm{Dr}$ Brandon Harvey of the NIDA Optogenetic and Transgenic Core for technical advice on their use. The opinions expressed in this article are the authors' own and do not reflect the view of the National Institutes of Health, the Department of Health and Human Services, or the United States government.

\section{References}

1. Association, A.P. . Diagonostic and statistical manual of mental disorders. American Psychiatric Publishing; Arlington, VA: 2013.

2. Moeller SJ, et al. Psychophysiological prediction of choice: relevance to insight and drug addiction. Brain. 2012; 135:3481-3494. [PubMed: 23148349]

3. Moeller SJ, et al. Functional, Structural, and Emotional Correlates of Impaired Insight in Cocaine Addiction. JAMA psychiatry. 2013

4. Goldstein RZ, et al. The neurocircuitry of impaired insight in drug addiction. Trends in Cognitive Sciences. 2009; 13:372-380. [PubMed: 19716751]

5. Barron HC, Dolan RJ, Behrens TE. Online evaluation of novel choices by simultaneous representation of multiple memories. Nature Neuroscience. 2013; 16:1492-1498. [PubMed: 24013592]

6. Takahashi YK, et al. Neural estimates of imagined outcomes in the orbitofrontal cortex drive behavior and learning. Neuron. 2013; 80:507-518. [PubMed: 24139047]

7. Abe H, Lee D. Distributed coding of actual and hypothetical outcomes in the orbital and dorsolateral prefrontal cortex. Neuron. 2011; 70:731-741. [PubMed: 21609828]

8. Stalnaker TA, et al. Orbitofrontal neurons infer the value and identity of predicted outcomes. Nature Communications. 2014 in press.

9. Daw ND, Niv Y, Dayan P. Uncertainty-based competition between prefrontal and dorsolateral striatal systems for behavioral control. Nature Neuroscience. 2005; 8:1704-1711. [PubMed: 16286932]

10. Huys QJ, et al. Bonsai trees in your head: how the Pavlovian system sculpts goal-directed choices by pruning decision trees. PLoS Computational Biology. 2012; 8:e1002410. [PubMed: 22412360]

11. Kalivas PW, Volkow ND. The neural basis of addiction: a pathology of motivation and choice. Am J Psychiatry. 2005; 162:1403-1413. [PubMed: 16055761]

12. Kalivas PW, O’Brien C. Drug addiction as a pathology of staged neuroplasticity. Neuropsychopharmacology. 2008; 33:166-180. [PubMed: 17805308]

13. Jentsch JD, Taylor JR. Impulsivity resulting from frontostriatal dysfunction in drug abuse: implications for the control of behavior by reward-related stimuli. Psychopharmacology. 1999; 146:373-390. [PubMed: 10550488]

14. Grimm JW, Hope BT, Wise RA, Shaham Y. Incubation of cocaine craving after withdrawal. Nature. 2001; 412:141-142. [PubMed: 11449260]

15. Epstein DE, Preston KL, Stewart J, Shaham Y. Toward a model of drug relapse: an assessment of the validity of the reinstatement procedure. Psychopharmacology. 2006; 189:1-16. [PubMed: 17019567]

16. Rescorla RA. Summation and overexpectation with qualitatively different outcomes. Animal Learning and Behavior. 1999; 27:50-62.

17. Takahashi $Y$, et al. The orbitofrontal cortex and ventral tegmental area are necessary for learning from unexpected outcomes. Neuron. 2009; 62:269-280. [PubMed: 19409271]

18. Wolf ME, Sun X, Mangiavacchi S, Chao SZ. Psychomotor stimulants and neuronal plasticity. Neuropharmacology. 2004; 47 (Suppl 1):61-79. [PubMed: 15464126] 
19. Kasanetz F, et al. Transition to addiction is associated wiht a persistent impairment in synaptic plasticity. Science. 2010; 328:1709-1712. [PubMed: 20576893]

20. Chen BT, et al. Rescuing cocaine-induced prefrontal cortex hypoactivity prevents compulsive cocaine seeking. Nature. 2013; 496:359-362. [PubMed: 23552889]

21. Crombag HS, Gorny G, Li Y, Kolb B, Robinson TE. Opposite effects of amphetamine selfadministration experience on dendritic spines in the medial and orbital prefrontal cortex. Cerebral Cortex. 2004; 15:341-348. [PubMed: 15269111]

22. Kasanetz F, et al. Prefrontal synaptic markers of cocaine addiction-like behavior in rats. Molecular Psychiatry. 2013; 18:729-737. [PubMed: 22584869]

23. Bowers MS, Chen BT, Bonci A. AMPA receptor synaptic plasticity induced by psychostimulants: the past, present and therapeutic future. Neuron. 2010; 67:11-24. [PubMed: 20624588]

24. Liu XB, Jones EG. Localization of alpha type II calcium calmodulin-dependent protein kinase at glutamatergic but not gamma-aminobutyric acid (GABAergic) synapses in thalamus and cerebral cortex. Proceedings of the National Academy of Science. 1996; 93:7332-7336.

25. Calu DJ, et al. Optogenetic inhibition of dorsal medial prefrontal cortex attenuates stress-induced reinstatement of palatable food seeking in female rats. Journal of Neuroscience. 2013; 33:214216. [PubMed: 23283335]

26. CMR, Bear MF. LTP and LTD: an embarrassment of riches. Neuron. 2004; 44:5-21. [PubMed: 15450156]

27. Mameli M, et al. Cocaine-evoked synaptic plasticity: persistence in the VTA triggers adaptations in the NAc. Nature Neuroscience. 2009; 12:1036-1041. [PubMed: 19597494]

28. Tan KR, et al. Neural bases for addictive properties of benzodiazepines. Nature. 2010; 463:769774. [PubMed: 20148031]

29. Yizhar O, Fenno LE, Davidson TJ, Mogri M, Deisseroth K. Optogenetics in neural systems. Neuron. 2011; 71:9-34. [PubMed: 21745635]

30. Kennerley SW, Behrens TE, Wallis JD. Double dissociation of value computations in orbitofrontal and anterior cingulate neurons. Nature Neuroscience. 2011 AOP.

31. Padoa-Schioppa C, Assad JA. Neurons in orbitofrontal cortex encode economic value. Nature. 2006; 441:223-226. [PubMed: 16633341]

32. Plassmann H, O’Doherty J, Rangel A. Orbitofrontal cortex encodes willingness to pay in everyday economic transactions. Journal of Neuroscience. 2007; 27:9984-9988. [PubMed: 17855612]

33. Gottfried JA, O’Doherty J, Dolan RJ. Encoding predictive reward value in human amygdala and orbitofrontal cortex. Science. 2003; 301:1104-1107. [PubMed: 12934011]

34. Schoenbaum G, Chiba AA, Gallagher M. Orbitofrontal cortex and basolateral amygdala encode expected outcomes during learning. Nature Neuroscience. 1998; 1:155-159. [PubMed: 10195132]

35. Tremblay L, Schultz W. Relative reward preference in primate orbitofrontal cortex. Nature. 1999; 398:704-708. [PubMed: 10227292]

36. Lucantonio F, Stalnaker TA, Shaham Y, Niv Y, Schoenbaum G. The impact of orbitofrontal dysfunction on cocaine addiction. Nature Neuroscience. 2012; 15:358-366. [PubMed: 22267164]

37. Volkow ND, Fowler JS. Addiction, a disease of compulsion and drive: involvement of orbitofrontal cortex. Cerebral Cortex. 2000; 10:318-325. [PubMed: 10731226]

38. Jentsch JD, Olausson P, De La Garza R, Taylor JR. Impairments of reversal learning and response perseveration after repeated, intermittent cocaine administrations to monkeys. Neuropsychopharmacology. 2002; 26:183-190. [PubMed: 11790514]

39. Franklin TR, et al. Decreased gray matter concentration in the insular, orbitofrontal, cingulate, and temporal cortices of cocaine patients. Biological Psychiatry. 2002; 51:134-142. [PubMed: 11822992]

40. Rogers RD, et al. Dissociable deficits in the decision-making cognition of chronic amphetamine abusers, opiate abusers, patients with focal damage to prefrontal cortex, and tryptophan-depleted normal volunteers: evidence for monoaminergic mechanisms. Neuropsychopharmacology. 1999; 20:322-339. [PubMed: 10088133] 
41. Bechara A, et al. Decision-making deficits, linked to a dysfunctional ventromedial prefrontal cortex, revealed in alcohol and stimulant abusers. Neuropsychologia. 2001; 39:376-389. [PubMed: 11164876]

42. Schoenbaum G, Setlow B. Cocaine makes actions insensitive to outcomes but not extinction: implications for altered orbitofrontal-amygdalar function. Cerebral Cortex. 2005; 15:1162-1169. [PubMed: 15563719]

43. Calu DJ, et al. Withdrawal from cocaine self-administration produces long-lasting deficits in orbitofrontal-dependent reversal learning in rats. Learning and Memory. 2007; 14:325-328. [PubMed: 17522022]

44. McCracken CB, Grace AA. Persistent cocaine-induced reversal learning deficits are associated with altered limbic cortico-striatal local field potential synchronization. Journal of Neuroscience. 2013; 33:17469-17482. [PubMed: 24174680]

45. Wied HM, Jones JL, Cooch NK, Berg BA, Schoenbaum G. Disruption of model-based behavior and learning by cocaine self-administration in rats. Psychopharmacology. 2013; 229:493-501. [PubMed: 23949256]

46. Goldstein RZ, Volkow ND, Wang GJ, Fowler JS, Rajaram S. Addiction changes orbitofrontal gyrus function: involvement in response inhibition. Neuroreport. 2001; 12:2595-2599. [PubMed: 11496155]

47. Bouton ME, Swartzentruber D. Sources of relapse after extinction in Pavlovian and instrumental learning. Clin Psychol Rev. 1991; 11:123-140.

48. Rescorla RA. Renewal after overexpectation. Learning and Behavior. 2007; 35:19-26. [PubMed: 17557388]

49. Rescorla RA. Spontaneous recovery from overexpectation. Learning and Behavior. 2006; 34:1320. [PubMed: 16786880]

50. Zhang F, Wang LP, Boyden ES, Deisseroth K. Channelrhodopsin-2 and optical control of excitable cells. Nature Methods. 2006; 3:785-792. [PubMed: 16990810] 


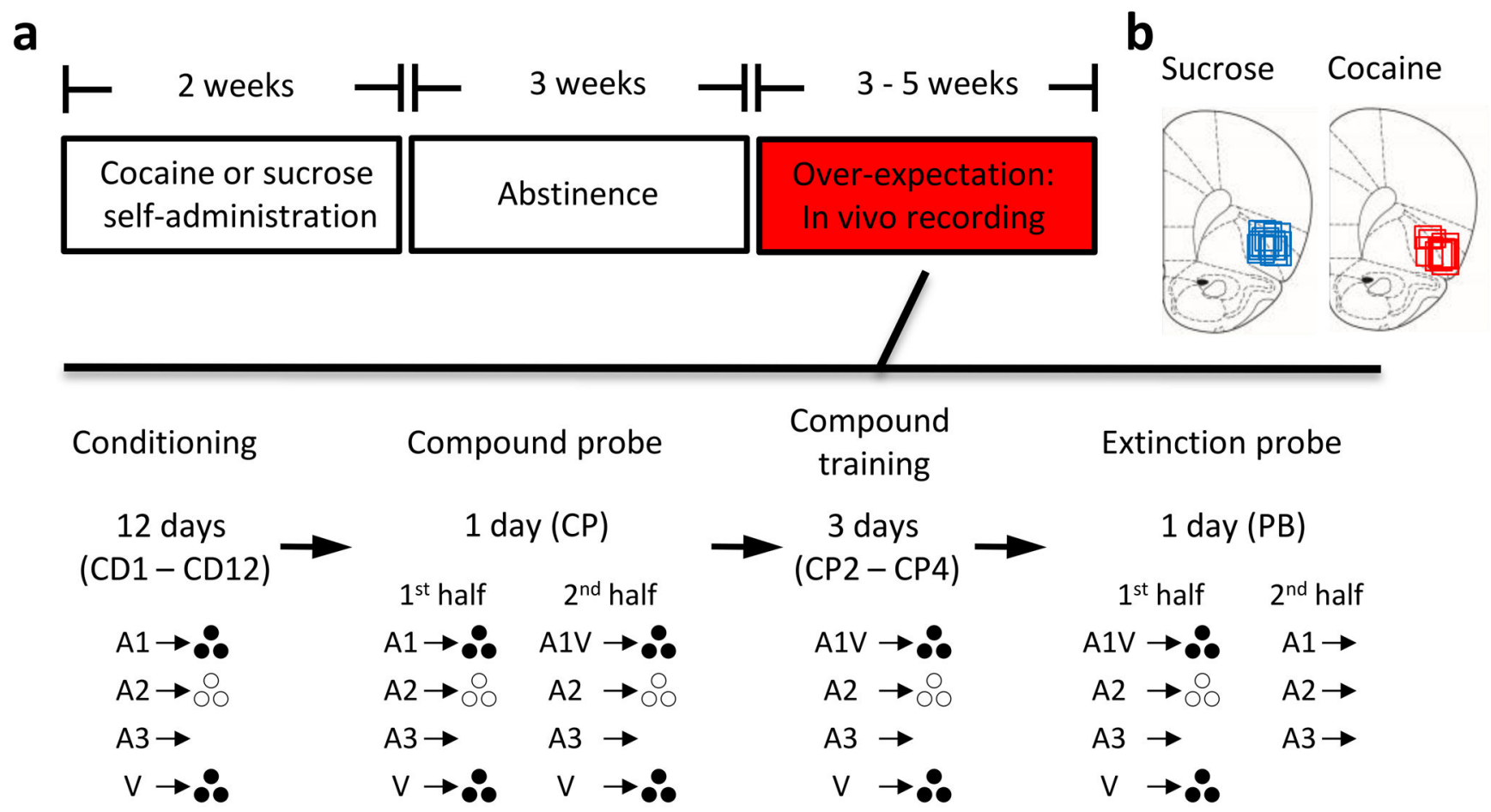

Figure 1. Experimental timeline, task design and recording sites for in vivo recording experiment

a. Timeline and task design. Approximately 3 weeks after the end of self-administration, rats were trained in a Pavlovian over-expectation task while single unit activity was recorded in OFC. b. Drawings illustrate recording sites in OFC in sucrose (blue) and cocaine-trained (red) rats. Boxes indicate approximate location of recording sites in each rat, taking into account any vertical distance traveled during training and the approximate lateral spread of the electrode bundle. 


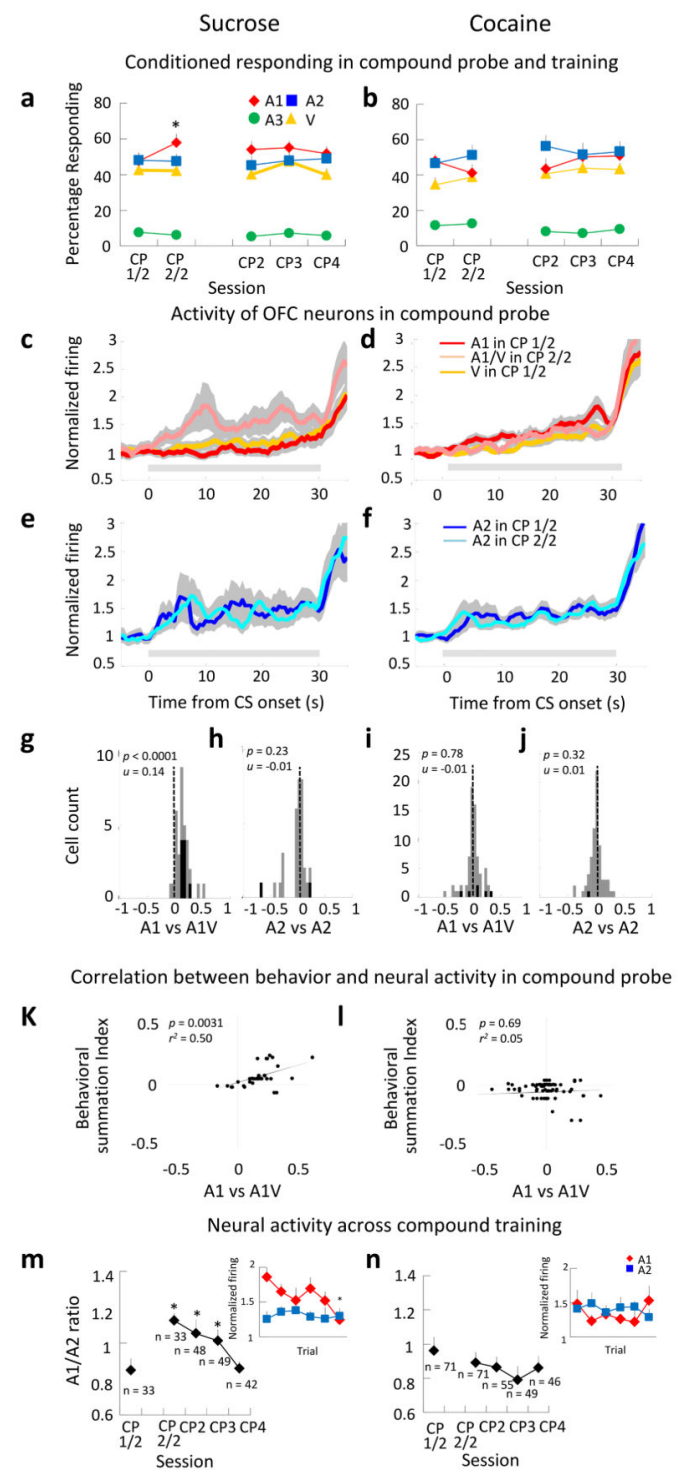

Figure 2. Conditioned responding and cue-evoked activity summates at the start of compound training in sucrose but not cocaine-trained rats

a-b. Conditioned responding in sucrose (a) and cocaine-trained (b) rats at the end of conditioning (CP 1/2) and through compound training (CP 2/2 and CP2-CP4). Error bars indicate S.E.M. A 3-factor ANOVA (cue X phase X treatment) showed a significant interaction between treatment, cue and phase $\left(\mathrm{F}_{1,18}=16.7, \mathrm{p}=0.0007\right)$, due to a significant increase in responding to $\mathrm{A} 1$ when it was paired with $\mathrm{V}$ in sucrose $(* ; \mathrm{p}<0.05)$ but not cocaine-trained (ns) rats. c-f. Population activity across all cue-responsive neurons to A1, V $(\mathbf{c}, \mathbf{d})$ and A2 (e, f) during the compound probe session; dark and light lines illustrate activity during the conditioning and compound phases of the session, respectively. Gray shading indicates S.E.M, and gray horizontal bars indicate the period of cue presentation. Two-factor ANOVA's (treatment X phase) showed significant effects of treatment on the pattern of firing to $\mathrm{A} 1\left(\mathrm{~F}_{1,102}=7.9, \mathrm{p}=0.0059\right)$ but not $\mathrm{A} 2\left(\mathrm{~F}_{1,102}=1.17, \mathrm{p}=0.28\right)$, due to a significant increase in firing to A1 in the sucrose but not the cocaine group at the start of 
compound training $(* ; p<0.05)$. $\mathbf{g}-\mathbf{j}$. Distribution of summation index scores for firing to A1 $(\mathbf{g}, \mathbf{i})$ and A2 $(\mathbf{h}, \mathbf{j})$ in the compound probe. Index scores were computed for each neuron based on the change in mean normalized firing to the relevant cue between conditioning and compound training, using the following formula: (firing CP $2 / 2$ - firing CP 1/2)/(firing CP $2 / 2+$ firing $\mathrm{CP} 1 / 2$ ). Black bars represent neurons in which the difference in firing was statistically significant ( $\mathrm{t}$-test, $\mathrm{p}<0.05$ ). In sucrose-trained rats, the distribution of the scores for A1 shifted significantly above zero and was significantly different from the unshifted distribution for A2; A1 also differed significantly between groups (Mann-Whitney U test, p's < 0.01). No shifts were observed in the scores from cocaine-trained rats. $\mathbf{k}-\mathbf{l}$. Scatter plots showing relationship between the change in behavior and neural activity to A1 in the compound probe session. Neural summation index scores were computed for firing to A1 as described above; behavioral summation index scores were computed similarly, for each session in which a cue-responsive neuron was recorded, but using conditioned responding instead of firing. Neural summation was correlated with behavioral summation in sucrose (k) but not cocaine-trained (l) rats. $\mathbf{m}-\mathbf{n}$. Line plots showing the ratio between normalized firing to $\mathrm{A} 1$ and $\mathrm{A} 2$ during each compound training session (CP - CP4). N's indicate number of cue-responsive neurons in each session. Error bars indicate S.E.M. A 2-factor ANOVA revealed a significant effect of treatment on the A1/A2 ratios $\left(\mathrm{F}_{4,412}=13.8, \mathrm{p}<\right.$ 0.0001 ), which increased significantly in the compound phase of the probe and then gradually decreased in sucrose (m) but not cocaine-trained rats (n). A similar effect was evident across trials within the compound probe session (inset, $\mathrm{F}_{5,505}=2.4, \mathrm{p}=0.036$ ). ${ }^{*} \mathrm{p}$ $<0.05$. 

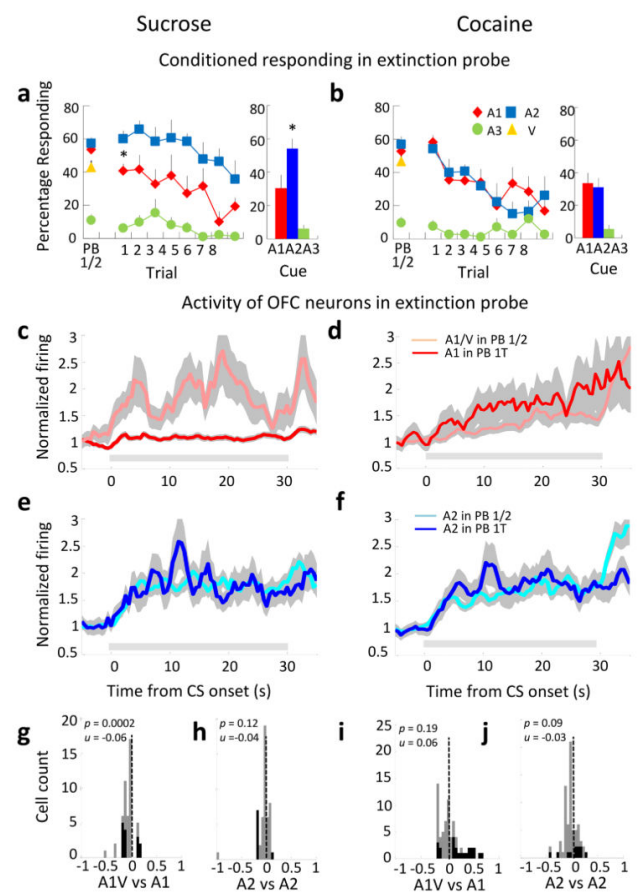

Correlation between behavior and neural activity in compound and extinction probe

$\mathbf{k}$

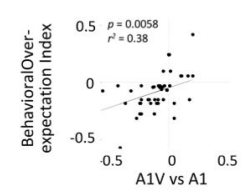

m

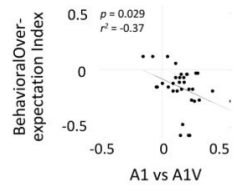

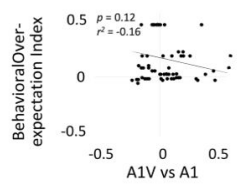

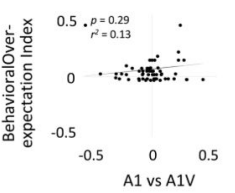

Figure 3. Conditioned responding and cue-evoked activity spontaneously declined at the start of extinction training in sucrose but not cocaine-trained rats

a-b. Conditioned responding in sucrose (a) and cocaine-trained (b) rats as a percentage of time in the food cup during each cue at the end of compound training (PB 1/2) and during the 8 trials of extinction (Trial 1-8 and bar graph showing means). Error bars indicate S.E.M., (*p < 0.05). A 3-factor ANOVA (cue X trial X treatment) revealed a significant interaction between treatment and cue $\left(\mathrm{F}_{2,36}=4.61, \mathrm{p}=0.016\right)$, due to a significant decline in responding to A1 when it was separated from V in sucrose $(* ; p<0.05)$ but not cocainetrained (ns) rats. Notably both groups showed extinction of responding to A1 and A2 across trials due to reward omission, with cocaine-treated rats showing somewhat more pronounced effects. c-f. Population activity across all cue-responsive neurons to A1, V(c, d) and A2 (e, f) during the extinction probe session; light and dark lines illustrate activity during the compound phase and on the first trial (1T) of extinction during the session, respectively. Gray shading indicates S.E.M, and gray horizontal bars indicate the period of cue presentation. Two-factor ANOVA's (phase X treatment) revealed significant effects of treatment on the pattern of firing to $\mathrm{A} 1\left(\mathrm{~F}_{1,118}=25.4, \mathrm{p}<0.0001\right)$ but not $\mathrm{A} 2\left(\mathrm{~F}_{1,118}=\right.$ 
$1.8, \mathrm{p}=0.17$ ) due to a significant decrease in firing to A1 in the sucrose but not the cocaine group at the start of extinction $(* ; p<0.05)$. $\mathbf{g}-\mathbf{j}$. Distribution of over-expectation index scores for firing to A1 $(\mathbf{g}, \mathbf{i})$ and A2 $(\mathbf{h}, \mathbf{j})$ in the extinction probe. Index scores were computed for each neuron based on the change in mean normalized firing to the relevant cue between compound training and the first trial of extinction, using the following formula: (firing PB $1 \mathrm{~T}$ - firing PB 1/2)/(firing PB $1 \mathrm{~T}+$ firing PB 1/2). Black bars represent neurons in which the difference in firing was statistically significant (t-test, $\mathrm{p}<0.05$ ). In sucrosetrained rats, the distribution of the scores for A1 shifted significantly below zero and was significantly different from the unshifted distribution for A2; A1 also differed significantly between groups (Mann-Whitney U test, p's $<0.01$ ). No shifts were observed in the scores from cocaine-trained rats. $\mathbf{k}-\mathbf{l}$. Scatter plots showing relationship between the change in behavior and neural activity to A1 on the first trial of extinction training. Neural overexpectation index scores were computed for firing to A1 as described above; behavioral over-expectation index scores were computed similarly, for each session in which a cueresponsive neuron was recorded, but using conditioned responding instead of firing. Neural changes were correlated with behavioral changes in sucrose (k) but not cocaine-trained (l) rats. $\mathbf{m}-\mathbf{n}$. Scatter plots showing relationship between the change in behavior on the first trial of extinction and neural activity to A1 at the start of compound training. Neural summation index scores were computed for firing to A1 as described in Figure 2. Neural summation was inversely correlated with behavioral over-expectation in sucrose (m) but not cocaine-trained $(\mathbf{n})$ rats. 
a

\begin{tabular}{|l|l|l|l|l|}
\hline $\begin{array}{l}\text { Cocaine or sucrose } \\
\text { self-administration }\end{array}$ & Abstinence & Over-expectation & Slice recording \\
\hline
\end{tabular}

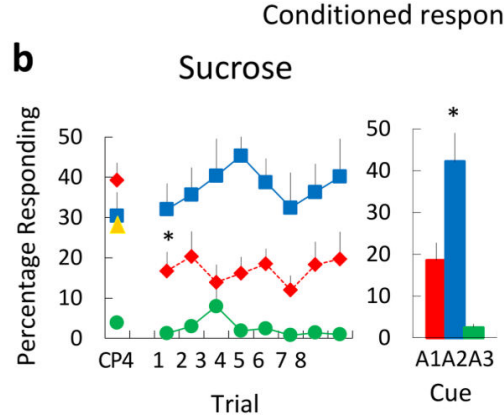

C

Cocaine

mEPSCs analysis

d

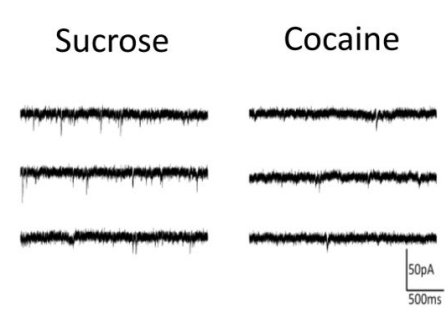

f
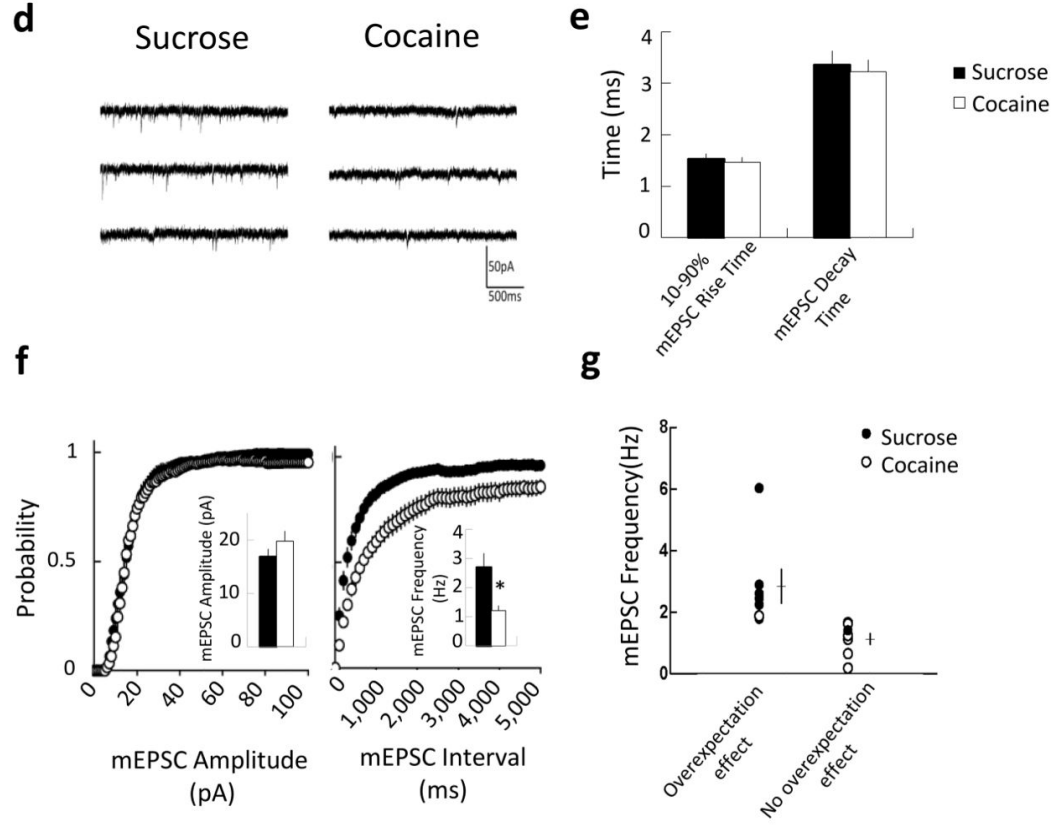

Figure 4. Reduced excitatory transmission in OFC pyramidal neurons in cocaine-trained rats a. Timeline for slice recording experiment. Approximately 3 weeks after the end of selfadministration, rats were trained in a Pavlovian over-expectation task illustrated in Figure 1. b-c. Conditioned responding in sucrose (b) and cocaine-trained (c) rats as a percentage of time in the food cup during each cue at the end of compound training (CP4) and during the 8 trials of extinction (Trial 1-8 and bar graph showing means). Error bars indicate S.E.M., (*p $<0.05$ ). A 3-factor ANOVA (cue X trial X treatment) revealed a significant interaction between treatment and cue $\left(F_{2,32}=5.65, p=0.008\right)$. Subsequent analyses showed that this was due to a significant decline in responding to $\mathrm{A} 1$ when it was separated from $\mathrm{V}$ in sucrose $(* ; p<0.05)$ but not cocaine-trained (ns) rats. d. Traces show pharmacologically isolated, mEPSCs recorded in OFC pyramidal neurons in brain slices from sucrose and cocaine-trained rats. e. Mean cumulative probability distributions for mEPSC amplitude and 
frequency for cells from sucrose ( $\mathrm{n}=26$ neurons, 9 rats) and cocaine-trained rats ( $\mathrm{n}=28$ neurons, 9 rats), showing a reduction in mEPSC frequency ( $p<0.0001, \mathrm{~K}-\mathrm{S}$ test). Insets: mean mEPSC parameters: amplitude $(* \mathrm{p}=0.0036, \mathrm{t}$ - test $)$ and frequency $(\mathrm{p}>0.05$, t-test). e. Mean mEPSC parameters: rise and decay times (p's $>0.05$, t-test). g. Rats that learned from over-expectation exhibited higher mEPSC frequencies ( $\mathrm{p}<0.05$, t-test). White and black circles indicate mean mEPSC frequency from individual rats included in the cocaine and sucrose-trained rats, respectively. The mean and s.e.m. of mEPSC frequency for these groups is also indicated by black and white horizontal and vertical lines, respectively. 
a

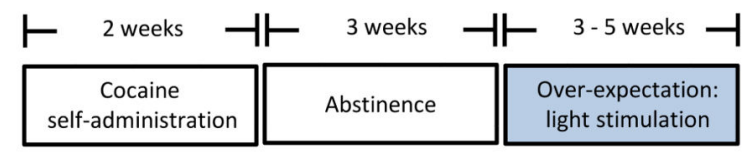

b

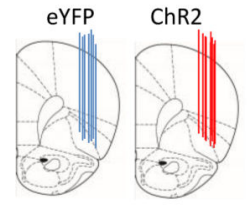

Optogenetic validation

Conditioned responding during the probe test

C
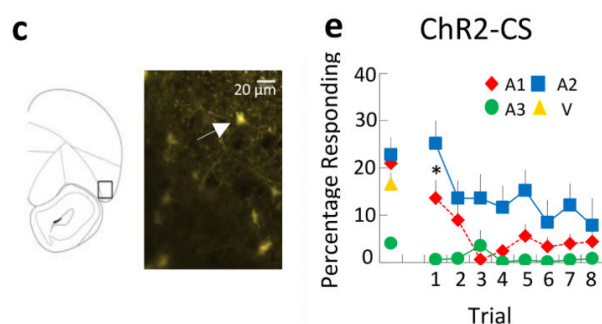

f $\quad \stackrel{P B}{1 / 2}$ eYFP-CS

d

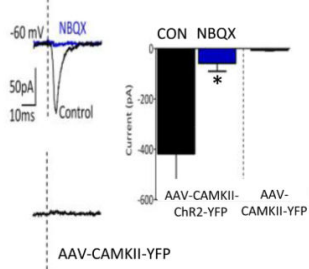

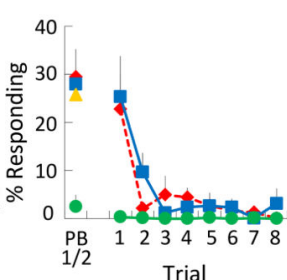

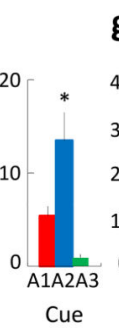

g ChR2-TI

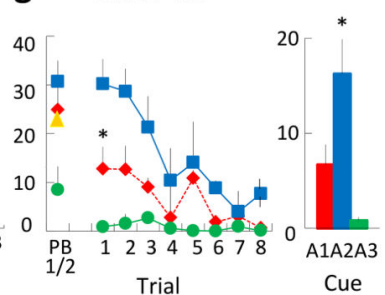

h $\quad$ EYFP-ITI
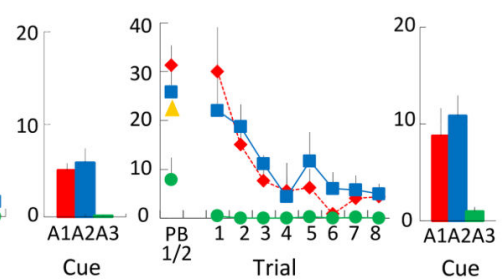

Figure 5. In vivo optogenetic activation of OFC neurons reverses the behavioral deficit in cocaine-trained rats

a. Timeline for the optogenetic experiment. Approximately 3 weeks after the end of selfadministration (7 weeks post-virus injection), rats were trained in a Pavlovian overexpectation task illustrated in Figure 1. b. Locations of cannula tracks in ChR2 (left) and eYFP (right) rats. c. left panel: approximate location (box) of confocal fluorescence image (right panel) showing expression of ChR2-eYFP (yellow) $\sim 8$ weeks after virus injection into OFC in a representative brain slice. d. Sample traces (left) and mean data (right) of synaptic currents evoked by a 5-ms $473 \mathrm{~nm}$ light pulse into the OFC in vitro, in AAV-CAMKIIChR2-YFP ( $n=4$ cells) and AAV-CAMKII-YFP $(n=4$ cells) injected rats. Light-evoked currents were only observed in the AAV-CAMKII-Chr2-YFP-injected group, and they were abolished by NBQX $(10 \mu \mathrm{M})$, an AMPAlkainate receptor antagonist. Dashed lines represent time of initiation of light stimulations. $\mathbf{e}-\mathbf{h}$. Conditioned responding in cocaine-trained rats as a percentage of time in the food cup during each cue at the end of compound training (PB 1/2) and during the 8 trials of extinction (Trial 1-8 and bar graph showing means) in ChR2CS (e), and eYFP-CS (f), ChR2-ITI (g), and eYFP-ITI (h) groups. Error bars indicate S.E.M., $(* \mathrm{p}<0.05)$. Three-factor ANOVA's (cue $\mathrm{X}$ trial $\mathrm{X}$ treatment) revealed significant interactions between treatment and cue for both $\mathrm{CS}\left(\mathrm{F}_{2,30}=3.84, \mathrm{p}=0.032\right)$ and ITI stimulation $\left(\mathrm{F}_{2,30}=4.02, \mathrm{p}=0.028\right)$. Subsequent analyses showed that this was due to significant declines in responding to A1 when it was separated from V in ChR2 (*; p < 0.05) but not eYFP (ns) rats. 